\title{
1 Prediction of the sex-determination gene in tunas (Thunnus fishes)
}

2

3 Yoji Nakamura ${ }^{1, *}, K^{*}$ taro Higuchi ${ }^{2, \dagger}$, Kazunori Kumon $^{3}$, Motoshige Yasuike ${ }^{1}$, Toshinori Takashi ${ }^{2}$,

$4 \quad$ Koichiro Gen ${ }^{2}$, Atushi Fujiwara ${ }^{4}$

5

$6 \quad{ }^{1}$ Bioinformatics and Biosciences Division, Fisheries Stock Assessment Center, Fisheries Resources

7 Institute, Japan Fisheries Research and Education Agency, 2-12-4 Fuku-ura, Kanazawa, Yokohama,

8 Kanagawa 236-8648, Japan

$9 \quad{ }^{2}$ Tuna Aquaculture Division, Aquaculture Research Department, Fisheries Technology Institute,

10 Japan Fisheries Research and Education Agency, 1551-8 Taira-machi, Nagasaki, 851-2213, Japan

$11{ }^{3}$ Amami Field Station, Tuna Aquaculture Division, Aquaculture Research Department, Fisheries

12 Technology Institute, Japan Fisheries Research and Education Agency, 955-5 Hyousakiyamahara,

13 Setouchi, Kagoshima 894-2414, Japan

$14{ }^{4}$ Aquatic Breeding Division, Aquaculture Research Department, Fisheries Technology Institute,

15 Japan Fisheries Research and Education Agency, 422-1 Nakatsuhamaura, Minami-ise, Mie,

16 516-0193, Japan

18 *Correspondence:

19 Email: yojnakam@affrc.go.jp (Y.N.); Tel. +81045 788 7673. Fax. +81 0457885001.

$20{ }^{\dagger}$ Current address: General Planning and Coordination Department, Headquarters, Japan Fisheries

21 Research and Education Agency, 1-1-25 Shin-urashima, Kanagawa-ku, Yokohama, Kanagawa

22 221-8529, Japan 


\section{$23 \quad$ Abstract}

24 Fish species have a variety of sex determination systems. Tunas (genus Thunnus) have an XY

25 genetic sex-determination system. However, the Y chromosome or responsible locus has not yet

26 been identified in males. In a previous study, a female genome of Pacific bluefin tuna (T. orientalis)

27 was sequenced, and candidates for sex-associated DNA polymorphisms were identified by a

28 genome-wide association study using resequencing data. In the present study, we sequenced a male

29 genome of Pacific bluefin tuna by long-read and linked-read sequencing technologies, and explored

30 male-specific loci through a comparison with the female genome. As a result, we found a unique

31 region carrying the male-specific haplotype, where a homolog of estrogen sulfotransferase gene was

32 predicted to be encoded. The genome-wide mapping of previously resequenced data indicated that,

33 among the functionally annotated genes, only this gene, named sult1st6y, was paternally inherited in

34 the males of Pacific bluefin tuna. We reviewed the RNA-seq data of southern bluefin tuna ( $T$.

35 maccoyii) in the public database and found that sult1st6y of southern bluefin tuna was expressed in

36 all male testes, but absent or suppressed in the female ovary. Since estrogen sulfotransferase is

37 responsible for the inactivation of estrogens, it is reasonable to assume that the expression of

38 sult1st6y in gonad cells may inhibit female development, thereby inducing the individuals to become

39 males. Thus, our results raise a promising hypothesis that sult st6y is the sex-determination gene in

40 Thunnus fishes, or at least functions at a crucial point in the sex-differentiation cascade.

42 Key words: bluefin tuna, sex determination, gene duplication, estrogen sulfotransferase 


\section{Introduction}

44 Fish species have a substantial variety of sex-determination systems compared to other animals,

45 ranging from environmentally to genetically modulated ones. In the environmental sex determination

46 system, individual sex depends mainly on nongenetic factors such as temperature, growth rate, and

47 density, while in the genetic system, specific genes dominantly control sex differentiation. Regarding

48 sexuality, it is estimated that more than $98 \%$ of fish are gonochoristic (Pandian, 2011). Therefore, in

49 most fish, some responsible loci should exist biasedly between males and females or differentially

50 affect gonadal phenotypes, even if the sex is primarily determined by environmental factors. In

51 heterogametic (XY) males or heterogametic (ZW) females, sex chromosomes may be cytologically

52 evident markers as carrying the sex determination loci, but they are often homomorphic in fishes

53 (Devlin and Nagahama, 2002), undistinguishable from autosomes by direct microscopic observation.

54 In other words, fish sex chromosomes are not highly differentiated, implying that turnovers may

55 have often occurred over the course of evolution (Pennell et al., 2018). Recently, sex determination

56 genes have been reported in several fish species, including DMY/Dmrt1 in medaka (Matsuda et al.,

57 2002; Nanda et al., 2002) and Chinese tongue sole (Cui et al., 2017), Amhr2 in fugu (Kamiya et al.,

58 2012), $s d Y$ in rainbow trout (Yano et al., 2012), Hsd17b1 in yellowtail (Koyama et al., 2019), and

59 amh in Patagonian pejerrey (Hattori et al., 2012) and northern pike (Pan et al., 2019). These studies

60 suggest that a master gene for sex determination in fish can be easily replaced by another gene,

61 which may be followed by the above-mentioned turnover of the sex chromosome. In particular, the

62 cases in fugu and yellowtail are prominent examples, where only single nucleotide substitutions are

63 critical for sex determination, suggesting that the replacements of master gene occurred only recently

64 in these lineages. Thus, the sex determination genes of fish are often different for each lineage and

65 are not always identified by deductive approaches from model organisms (that is, case studies are

66 required to identify the genes). 
Regarding tunas, the fish of the genus Thunnus South 1845, it is estimated that Pacific bluefin tuna (Thunnus orientalis, PBT) has an XY genetic sex determination system (Agawa et al., 2015). However, the chromosome or locus responsible has not yet been identified. One reason for this is that no distinguishable sex chromosomes were observed in the cytological analysis. It has been reported that little tuna, Euthynnus affinis, may have a ZW sex determination system (Yazawa et al., 2019). These observations suggest that the sex determination gene of Thunnus species has only recently emerged, and therefore, that the sex chromosomes are not highly divergent. Another reason is the technical difficulty in handling: since tunas are large and difficult fish to culture, in vivo experiments for identifying sex-associated loci are costly and time-consuming. On the other hand, because tunas have a high market value worldwide, tuna target fishing and aquaculture have been conducted in many countries. In aquaculture, the control of the sex ratio in tanks or ocean nets is very important for breeding, as keeping more females than males leads to an increased production of fertilized eggs. Since tunas do not show any clear sexual dimorphism, the direct observation of the gonad has been the main technique for sex identification. However, this is a destructive inspection and is not applicable for selectively collecting females or removing males in tanks. Therefore, molecular markers associated with the sex of tuna have been of interest as an alternative for rapid

83 sex identification. In particular, DNA markers have been considered promising, where a small

84 portion of tissue sections, such as the fin or skin, are sufficient for PCR assays. A DNA marker associated with PBT sex was reported in 2015 (Agawa et al., 2015). The marker, Md6, was developed from the DNA samples of cultured PBT individuals and showed an association with sex in the aquaculture population. Regarding the genomic approach, the PBT male genome data were open (Nakamura et al., 2013), but the scaffold sequences were too fragmented for a genome-wide

89 screening of sex-associated loci. Recently, transcriptomics of southern bluefin tuna (T. maccoyii)

90 have been conducted, and the differentially expressed genes between male and female gonads (testis 
and ovary) were examined (Bar et al., 2016). However, it should be noted that a differentially

expressed gene between the testis and ovary is not always the sex determination gene, but may be a downstream gene regulated by the master gene.

In 2019, Suda et al. sequenced the genome of female PBT, compared the assembled scaffold scaf64_3726411_F/R, and scaf64_3724591_F/R, based on male-specific heterozygous SNVs and short indels detected in the 6.5-kb region of female scaffold 64 (F64). The PCR primers were designed so that the amplification patterns were different between PBT males and females: scaf64_3724604_F/R and scaf64_3726411_F/R were amplifiable in males (the sizes were $113 \mathrm{bp}$ and 143 bp, respectively) but not in females, and scaf64_3724591_F/R was amplified in both males and females, but the sizes were different (142 bp and 149 bp in males and only 149 bp in females). PCR amplification using 115 individuals (56 males and 59 females) yielded $100 \%$ accuracy of sex identification, implying that the PBT sex-determination gene may exist around the 6.5-kb region in F64. They predicted some genes in F64 and discussed the possible involvement in the sex determination of PBT. However, promising genes, such as those related to the cascade, were not identified. The potential problem in the previous study was that they found male-specific

111 heterozygous polymorphisms in multiple scaffolds. Since most sex determinants are often attributed to a single locus, they suspected that multiple candidates occurred due to assembly errors. 
bioRxiv preprint doi: https://doi.org/10.1101/2021.06.15.448492; this version posted June 29, 2021. The copyright holder for this preprint (which was not certified by peer review) is the author/funder. All rights reserved. No reuse allowed without permission.

115 involved in estrogen inactivation. The current results provide the most promising candidate for the 116 sex determination gene of tuna. 


\section{Materials and Methods}

\section{Genome sequencing and assembly}

119 A three-year-old male F2 individual of Pacific bluefin tuna, which was cultured at the Seikai

120 National Fisheries Research Institute (currently Fisheries Technology Institute), was collected for

121 genome sequencing. Frozen heart tissue was prepared from the specimen, and DNA was extracted

122 using a DNeasy Blood \& Tissue Kit (Qiagen, Venlo, Netherlands). Then, an SMRT-bell template

123 library was prepared following the manufacturer's protocol and sequenced using the Pacific

124 Biosciences (PacBio) Sequel platform (P6C4 chemistry) (Pacific Biosciences, Inc. CA). From the

125 same tissue sample, the DNA was extracted also using the standard phenol-chloroform protocol. A

126 Chromium 10X library was prepared using Chromium Genome Library Kit v2, Genome Gel Bead

127 Kit, Genome Chip Kit v2, and i7 Multiplex Kit (10X Genomics, CA) following the manufacturer's

128 protocol, and the linked reads were sequenced using the Illumina NovaSeq 6000 platform (Illumina,

129 Inc. CA). The PacBio reads were first assembled by HGAP4 (SMRT Link v5.1.0.26412) (Chin et al.,

130 2013). The contigs obtained were corrected using NovaSeq linked-reads by Pilon v1.23 (Walker et

131 al., 2014) and Tigmint v1.1.2 (Jackman et al., 2018), and finally scaffolded by ARKS v1.0.2

132 (Coombe et al., 2018) in combination with LINKS v1.8.5 (Warren et al., 2015). The scaffold

133 sequences obtained were submitted to the DNA Data Bank of Japan under the accession nos.

134 BOUD01000001-BOUD01000948. To examine the phased genomic regions, the linked reads were

135 assembled using Supernova2 (v2.1.1) (Weisenfeld et al., 2017). The former assembly (HGAP4 +

136 ARKS) was assessed by Benchmarking Universal Single-Copy Orthologs (BUSCO) version 4.0.5

137 (Simão et al., 2015) with a set of Actinopterygii orthologs (actinopterygii_odb10). The genes around

138 the sex-associated region were predicted based on the protein sequences of model fish species in the

139 Ensembl database (release 99) (Aken et al., 2017), namely Danio rerio, Oryzias latipes,

140 Gasterosteus aculeatus, Takifugu rubripes, and Tetraodon nigroviridis, by Exonerate v2.4.0 (Slater 
141 and Birney, 2005). The PBT female genome sequence was obtained from the GenBank database

142 (accession no. BKCK00000000). The nucleotide sequence comparison was performed by MUMmer

143 v4 (Marcais et al., 2018). The repeat library of PBT genome was constructed by RepeatModeler

144 Open-1.0, and the repetitive regions were masked by RepeatMasker Open-4.0

145 (http://www.repeatmasker.org). The duplicated regions were further detected by self-to-self

146 BLASTN (Altschul et al., 1990) in each of the PBT male and female genomes, and the sequences

147 detected were used also for masking.

\section{Genome-wide SNV/CNV analysis}

150 The sequenced reads for 31 PBT individuals (15 males and 16 females) (Suda et al., 2019) were

151 downloaded from the NCBI SRA database (accession no. DRR177387). The read sequences were

152 extracted using SRA Toolkit (https://trace.ncbi.nlm.nih.gov/Traces/sra/sra.cgi?view=software), and

153 then preprocessed using Trimmomatic v0.36 (Bolger et al., 2014) and ParDRe v2.2.5

154 (Gonzalez-Dominguez and Schmidt, 2016), according to Suda et al. Mapping to the scaffold

155 sequences and single-nucleotide variant calling were conducted using MapCaller v0.9.9.37 (Lin and

156 Hsu, 2019). After filtering out inadequate sites (not biallelic, depth < 20), genome-wide association

157 (GWA) between males and females was estimated through $2 \times 3$ contingency tables using the R

158 package, $\operatorname{rrBLUP}$ (Endelman, 2011), with minor allele frequency $>0.05$. Statistical significance was

159 defined as $P<0.05$ after Bonferroni correction based on the total number of variant sites examined.

160 For relative depth analysis, raw read depth per nucleotide site was counted using Samtools (samtools

161 depth) (Li et al., 2009), and then divided by the median depth. Copy number variations (CNVs) were

162 detected by CNVcaller (Wang et al., 2017), where the above-mentioned mapping data were scanned

163 by a 500-bp window and regional copy numbers were obtained by merging them in the neighboring

164 windows. The difference in the regional copy number between males and females was tested using 
165 the Mann-Whitney $U$ test with Bonferroni correction. Furthermore, the regions carrying either of the

166 three types of copy numbers $(0,0.5$, or 1$)$ among the 31 samples were selected, and the GWA was

167 estimated in the same way as the SNV-based GWA scan mentioned above.

\section{Sequence analysis of sex-associated genes}

170 The transcriptome data of southern bluefin tuna (Bar et al., 2016) were downloaded from the NCBI

171 SRA database (accession no. SRP059929). The read sequences were extracted using SRA Toolkit,

172 preprocessed by Trimmomatic, and assembled using Trinity v2.8.4 (Haas et al., 2013). From the

173 contig sequences obtained, protein-coding sequences were predicted using TransDecoder v5.0.0

174 (Haas et al., 2013). Homologs of PBT genes were searched using BLASTP (Altschul et al., 1997)

175 from the contig sequences, and the transcriptional variants were manually checked based on the

176 sequence alignment using MAFFT v7.310 (Katoh et al., 2009). Using the Trinity contig sequences as

177 a reference, gene expression was measured using RSEM v1.3.1 (Li and Dewey, 2011). For molecular

178 phylogenetic analysis, homologs in other fish genomes were downloaded from the Ensembl database.

179 The coding sequences were then aligned using MAFFT, where the deduced protein sequences were

180 aligned and converted into nucleotide sequence alignments. The maximum-likelihood tree was

181 constructed using RAxML-NG v0.9.0 (Kozlov et al., 2019). 


\section{Results}

\section{Genome assembly and validation of sex-associated markers}

184 We constructed the genome sequence of male PBT by de novo assembly, yielding a total of 948

185 scaffolds (>1000 bp) and totaling $827 \mathrm{Mb}$ (Table 1). Using BUSCO for quality assessment, this

186 assembly completely captured $96.3 \%$ of Actinopterygii orthologs (3505 out of 3460). On the other

187 hand, the score for the female genome was $89.3 \%$ (3253 out of 3640), consistent with previous

188 reports. Using the sex-associated PCR primer sequences reported in a previous study, we performed

189 a BLAST search against the current male genome, and found that two scaffolds, namely M175 and

190 M44, carried the regions amplifiable by the primer sets (two male-specific sets,

191 scaf64_3724604_F/R and scaf64_3726411_F/R, and a common primer set, scaf64_3724591_F/R)

192 (Table 2). In scaffold M175 (232,647 bp in total), the regions amplified by the three primer sets were

193 closely located. The estimated amplicon sizes were congruent with those previously observed only in

194 males: 113 bp for scaf64_3724604_F/R, 143 bp for scaf64_3726411_F/R, and 142 bp for

195 scaf64_3724591_F/R, respectively. Scaffold M44 (4,583,724 bp in total) had a single region

196 amplified by scaf64_3724591_F/R, and the estimated amplicon size was 149 bp, which was

197 equivalent to that observed previously in both males and females. Thus, based on the estimated PCR

198 products, M175 carried all of the male-specific regions and M44 carried the common haplotype to

199 both males and females. We compared the nucleotide sequences of M175 and M44, and found that

200 M175 was almost dissimilar to that of M44 (Figure 1), while M44 was fully aligned with female

201 scaffold F64 (Figure 2), in which the 6.5-kb sex-linked region (SLR) was reported in the previous

202 study. The 6.5-kb SLR was found also in M44. On the other hand, we found no counterpart of M175

203 in the female genome, although the 6.5-kb SLR of M44/F64 (SLR $\mathrm{M} 44_{4}$ or $\left.\mathrm{SLR}_{\mathrm{F} 64}\right)$ was partially

204 similar to the 10.7-kb region in M175 ( $\operatorname{SLR}_{\mathrm{M} 175}$ ) (Figure 1). We assembled the male genome using

205 Supernova2 and obtained scaffolds corresponding to M44 and M175, respectively (Supplementary 
206 Figure 1). The M44-like scaffold carrying SLR $_{\mathrm{M} 44}$ was phased into two pseudo-haploid scaffolds,

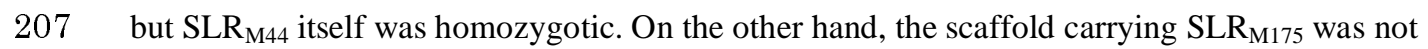
208 phased.

\section{$210 \quad$ Read depth analysis}

211 We downloaded the resequencing data of 31 PBT individuals (15 males and 16 females) and mapped

212 the reads to the current male genome sequence. As a result, both male and female reads were fully

213 mapped to SLR $_{\mathrm{M} 44}$ (Figure 3 and Supplementary Figure 2). Regarding M175, the female reads were

214 hardly mapped to $\mathrm{SLR}_{\mathrm{M} 175}$, in contrast to the male reads. Furthermore, there were consistently fewer

215 male reads mapped to SLR $_{\mathrm{M} 175}$ than SLR $_{\mathrm{M} 44}$. We computed the median read depths for whole

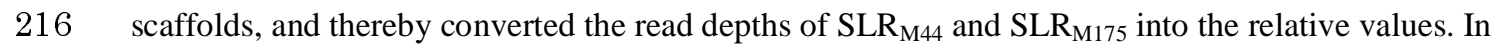

217 males, the relative depths of SLR M175 $_{1}$ were close to 0.5 (Figure 4). On the other hand, the relative

218 depths of SLR $\mathrm{M}_{44}$ were close to 1 in both males and females. These observations strongly suggest

219 that, in shotgun sequencing libraries, the DNA content derived from SLR M175 $_{1}$ in the male samples is

220 always half that derived from the average scaffolds, reflecting the ploidy or copy number. The

221 majority of chromosomal regions, except for highly repetitive ones or those of polyploidized

222 genomes, should exist as singletons in a diploid manner (2n), and the relative depths for many

223 regions would be approximately 1 . Therefore, our observations suggest that SLR $\mathrm{M}_{175}$, with a relative

224 depth of $\sim 0.5$, should exist in a haploid manner $(1 \mathrm{n})$ only in males, while SLR $\mathrm{M}_{44}$ should exist in a

225 diploid manner, like many other regions, in both males and females.

\section{Genome-wide association analysis}

228 Since the results obtained until now appeared to be inconsistent with those previously reported (see

229 Discussion), we validated the previous mapping and GWA results based on the female genome. We 
230 mapped the 31 resequenced read sets to the female reference genome and re-performed GWA

231 analysis. As a result, the previous result was roughly reproduced, with significant peaks observed in 232 multiple scaffolds, including F64 (Figure 5A). However, when the mapped reads between the female 233 and present male genomes were compared, over $10 \%$ of the reads mapped to female SLR $\mathrm{F} 64_{4}$ in the

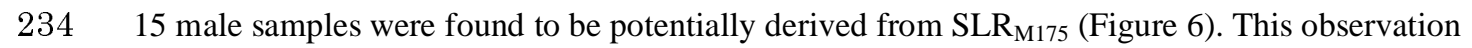

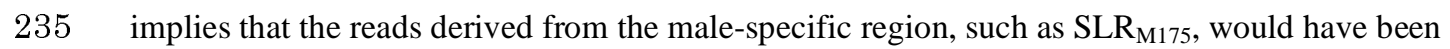
236 incorrectly mapped to paralogous regions in the female genome in the previous study. To avoid the 237 effect of such cross mapping, we performed the GWA scan again after the duplicated regions were 238 thoroughly masked by RepeatMasker and BLASTN. This result was in contrast to that from the 239 unmasked reference: no significant peaks were observed in the masked sequences (Figure 5B).

240 Similarly, GWA scans were performed using the current male genome, but no significant peaks were 241 found in the masked sequences (Supplementary Figure 3).

242 We then tested another approach to identify the regions carrying male-specific haplotypes, such 243 as $\mathrm{SLR}_{\mathrm{M} 175}$. Using the mapping data for the 31 individuals, we computed copy number variations 244 (CNVs) across the male genome and explored the regions whose copy numbers were significantly 245 different between males and females. As a result, only one candidate was found in M175 $246(18,001-78,750 \mathrm{bp})$, very close to $\mathrm{SLR}_{\mathrm{M} 175}$, and the copy number in males was significantly larger 247 than that in females (Figure 7A). All of the males had 0.5 copies of the detected region and all of the 248 females had no copy (i.e., absent), in concordance with the relative depth analysis results (Figure 4).

249 We computed genome-wide associations for the haploid regions by converting the copy numbers $(0$,

$250 \quad 0.5$, and 1$)$ to three quasi-genotypes $(0 / 0,0 / 1$, and $1 / 1)$. As a result, only one significant peak for this 251 region was detected in scaffold M175 (Figure 7B). 
254 We scanned the M175 sequence and predicted 12 protein-coding genes (Figure 8 and Supplementary

255 Table 1). Of these, two genes, g3 (32,614-64,628 bp) and g4 (76,852-77,963 bp), were located in

256 the haploid region (18,001-78,750 bp) detected by CNV analysis. The deduced amino acid sequence

257 of g3 was similar to that of estrogen sulfotransferase encoded by sult1st6 (e.g., with $\sim 83 \%$ identity to

258 ENSORLP00000007539 of Oryzias latipes). Therefore, the homolog of PBT was named sult1st6y.

259 In the case of $\mathrm{g} 4$, the deduced amino acid sequence was similar to that of ENSTRUP00000072273

260 (gene ID: ENSTRUG00000033234) of Takifugu rubripes, with 70\% identity. As a "novel gene" in

261 the Ensembl database, the function was unknown. However, the domain "Integrase, catalytic core"

262 was predicted by Pfam and PROSITE, and found to overlap with the Gene3D domain "Ribonuclease

263 H superfamily." With regard to the neighboring genes, the protein sequence of g2 was matched to

264 ENSTRUP00000068256 (ENSTRUG00000032453, novel gene), with the Pfam domain "PiggyBac

265 transposable element-derived protein,” and g5 was matched to ENSTRUP00000067783

266 (ENSTRUG00000032600, novel gene) with the Pfam domain "Reverse

267 transcriptase/retrotransposon-derived protein, RNase H-like domain.” Notably, g1 was similar to g5

268 and matched to ENSTRUP00000067783, suggesting that g2-g4 were sandwiched between

269 duplicated genes (g1 and g5). In addition, g6 was matched to ENSORLP00000040498

270 (ENSORLG00000025541, novel gene) with the Pfam domain “Transposase, Tc1-like," and the other

271 genes, g7-g12, were matched to the same protein, ENSORLP00000044417

272 (ENSORLG00000028531, novel gene) with the Gene3D domain "Immunoglobulin-like fold." Thus,

273 only g3 was a functionally annotated gene, and the others were novel or related to transposable

274 elements. We found no homologs of sult1st6y (g3) in the scaffolds M44/F64. Instead, the paralog

275 was found in male scaffold 30 (M30, 6594208-6602041 bp), named sult1st6a, and the nucleotide

276 and protein identities to sult 1 st $6 y$ were $92 \%$ and $89 \%$, respectively. Male scaffold M30 corresponded

277 to a part of female scaffold 47 (F47) (Supplementary Figure 4), where sult1 st6a was also found. 
278 To determine whether these sult1st6 genes are present in other tuna species, we downloaded the

279 RNA-seq data of southern bluefin tuna (SBT), which were obtained from 10 male testis samples and

28010 female ovary samples. We assembled the reads using Trinity and conducted a homology search

281 against the contigs using PBT sult1st6y/sult1st6a sequences. As a result, we found the homologs of

282 sult st6y/sult1st6a in SBT, although the Trinity contigs were originally classified into a single cluster.

283 We conducted phylogenetic analysis using PBT and SBT sult1st6 and the homologs in the Ensembl

284 database (Figure 9 and Supplementary Figure 5). The phylogenetic tree showed that each SBT

285 homolog formed a clade with each of PBT's sult1st6y/sult1st6a, respectively, suggesting that SBT

286 also had two paralogs of sult1st6 and the duplication to sult1st6y and sult1st6a occurred in the

287 common ancestor of PBT and SBT. Using the transcript contigs as a reference, we mapped the reads

288 in each of the 20 samples to those and measured the gene expression levels (Figure 10). As a whole,

289 the expression of SBT's sult1st6y/sult1st6a was low compared to that of the control (0-2\% of $\beta$-actin

290 in FPKM). In detail, the expression of sult1 st6a was observed in both the testis and ovary samples,

291 although it tended to be low in the latter (0-0.01\% of $\beta$-actin in FPKM) (Figure 10A). The

292 expression of sult1st6y was observed in all 10 testis samples, but none of ovary samples, indicating a

293 significant bias of sult1st6y expression between the testes and ovaries $(P<0.05$, Fisher's exact test $)$

294 (Figure 10B). 


\section{Discussion}

296 In this study, the male genome of Pacific bluefin tuna was sequenced using long-read

297 sequencing technologies. The scaffold sequences assembled were more contiguous than those

298 published in 2013, which were obtained from pyrosequencing and short paired-end reads.

299 Completeness assessment indicates that the current version is better than that of the recent female

300 genome, although the number of scaffolds (948) is larger than that for the female genome (444).

301 Therefore, the current genome is expected to be useful for the validation and extension of previous

302 results regarding the sex identification of PBT. In the previous study, three PCR primer sets were

303 designed from the sequence of female scaffold F64, so that the amplification patterns were different

304 between PBT males and females. In this study, we first searched for the locus amplified by the

305 primer sets in the current male genome, and found two regions: one in scaffold M44 and the other in

306 M175. Based on the results of the large-scale sequence comparison, M44 was found to be the

307 counterpart of F64, while M175 was male-specific. Since M44 and F64 were well aligned with each

308 other, including 6.5-kb SLRs ( $\left.\mathrm{SLR}_{\mathrm{M} 44} / \mathrm{SLR}_{\mathrm{F} 64}\right)$, it is unlikely that large-scale misassemblies are

309 present in M44/F64. The PCR amplification pattern in $\mathrm{SLR}_{\mathrm{M} 44}$, estimated using primer sequences,

310 was identical to that observed in females. Regarding M175, all the PCR amplicons with

311 male-specific sizes were observed within the 10.7-kb region $\left(\mathrm{SLR}_{\mathrm{M} 175}\right)$. It is worth noting that the

312 PCR primer sets were originally designed based on the SNVs predicted in F64 by GWA analysis.

313 Therefore, according to the previous results, M44 and M175 should be allelic to each other. However,

314 these sequences are quite dissimilar to each other. Furthermore, based on the mapping result of

315 resequenced data, the relative read depths to that for the average scaffold data were $\left(\mathrm{SLR}_{\mathrm{M} 44}\right.$,

$\left.316 \operatorname{SLR}_{\mathrm{M} 175}\right) \approx(1,0)$ in females but $\left(\operatorname{SLR}_{\mathrm{M} 44}, \mathrm{SLR}_{\mathrm{M} 175}\right) \approx(1,0.5)$ in males. These estimates indicate that

317 SLR $_{\mathrm{M} 44}$ should exist in a diploid manner in both males and females, whereas SLR $_{\mathrm{M} 175}$ should exist in

318 a haploid manner only in males. If $\mathrm{SLR}_{\mathrm{M} 44}$ was allelic to $\mathrm{SLR}_{\mathrm{M} 175}$ in males, the relative read depths 
$319\left(\mathrm{SLR}_{\mathrm{M} 44}, \mathrm{SLR}_{\mathrm{M} 175}\right)$ should be $(0.5,0.5)$. The result of relative read depth is congruent with that of

320 phased assembly by Supernova2, where two pseudo-haplotype scaffolds carrying homozygotic

321 SLR $_{\mathrm{M} 44}$ were obtained, but only unphased one was obtained for SLR $\mathrm{M}_{175}$. These results indicate that

322 SLR $_{\mathrm{M} 44}$ and $\mathrm{SLR}_{\mathrm{M} 175}$ are located at different loci in the male genome, where SLR $\mathrm{M}_{175}$ is the

323 male-specific structural variant (i.e., inserted in males or deleted in females) and is inherited

324 paternally. In other words, $\mathrm{SLR}_{\mathrm{F} 64}$ (or $\mathrm{SLR}_{\mathrm{M} 44}$ ) reported in the previous study was not associated

325 with PBT sex, although the PCR primer sets designed from SLR $_{\mathrm{F} 64}$ are still available for sex

326 identification based on the amplification pattern in SLR $_{\mathrm{M} 175}$.

327 Now, the previous result, in which male-specific heterozygous polymorphisms were detected in

328 F64, needs to be reconsidered. The previous GWA scan was performed using the female genome as a

329 reference, focusing on male-heterozygous SNVs or small indels, which may be detectable by the

330 resequenced short reads. However, M175 found in the current study is a large-scale structural variant

331 absent from the female genome and should be undetectable by short-read mapping. We considered

332 the possibility that the reads derived from paralogous loci were cross-mapped to the female reference

333 genome, causing fake heterozygous SNV calls in genotyping prior to GWA analysis. In the previous

334 study, the repeat or paralogous sequences were not masked; therefore, it is likely that the reads

335 derived from the paternal $10.7-\mathrm{kb}$ region (i.e., $\mathrm{SLR}_{\mathrm{M} 175}$ ) were mapped to the 6.5-kb region of F64 in

336 the analysis of male DNA samples. This hypothesis was confirmed by checking the mapped reads: at

337 least $10 \%$ of the male reads mapped to $\mathrm{SLR}_{\mathrm{F} 64}$ in the previous analysis were estimated to be derived

338 from SLR $_{\mathrm{M} 175}$. Furthermore, we compared the GWA results between the two conditions for the

339 female reference sequence: duplicated regions were unmasked (i.e., raw sequences) and masked. The

340 result was clear-cut, where the peaks in multiple scaffolds were roughly reproduced in the unmasked

341 sequences, but no such peaks were observed in the masked sequences. Thus, we conclude that the

342 previous GWA result was an artifact due to cross-mapping, and many of the scaffolds predicted (e.g., 
343 F64) were not associated with the sex of PBT. Since we found no significant GWA peaks in the

344 masked male reference genome, the SLR of PBT would be undetectable from the information of

345 SNVs or small indels. The current results provide a caveat that traditional GWA analysis, which

346 targets SNVs using short reads, may not always be sufficient for the identification of SLR in species

347 with a genetic sex determination system.

348 Instead of $\mathrm{SLR}_{\mathrm{M} 44}$, we found that $\mathrm{SLR}_{\mathrm{M} 175}$ is a male-specific locus absent from the female

349 genome. It should be noted that this finding is due to a homology search of the primer sequences

350 designed in the previous study. Therefore, it is possible that the current male genome contains other

351 scaffolds carrying sex-associated structural variants. To check this possibility, we conducted a

352 genome-wide scan based on a CNV detection scheme using mapping data. As a result, we observed

353 that only M175 had a 61-kb region (18,001-78,750 bp) where the CNV was significantly different

354 between males and females. Since this region is very close to SLR $_{\mathrm{M} 175}$, eventually the candidate

355 sex-associated locus was narrowed down to a single region around or including SLR $_{\mathrm{M} 175}$. Regarding

356 the CNV detected, we found that PBT females have no copy, while males have 0.5 copies of the

357 region, indicating that this region exists in a haploid manner in males, or equivalently, that this

358 region is paternally inherited. Thus, we conclude that this region (61-kb region + SLR $\left.\mathrm{M}_{\mathrm{M} 15}\right)$ is the

359 only candidate for the Y-linked locus in PBT. In M175, we predicted a total of 12 protein-coding

360 genes, two of which (g3 and g4) were included in the predicted haploid region, one (g3) of which

361 was annotated as encoding estrogen sulfotransferase (SULT1ST6), while the other (g4) was

362 functionally unknown. We note that g4 has the domain "Integrase, catalytic core" or "Ribonuclease

363 H superfamily" which is a feature of the retroviral integrase superfamily (Nowotny, 2009).

364 Regarding the other 10 predicted genes in M175, the functions are unknown. In particular, g1, g2,

365 and g5 may be involved in transposons according to domain prediction. Therefore, around the

366 male-specific haploid region, only the centermost g3, namely sult1st6y, is functionally annotated and 
sandwiched between possibly transposable elements, implying that the g3 locus may have been unstable and transposed from the ancestral locus. cytosolic sulfotransferases (SULTs) and has been studied as a detoxifying enzyme in mammals (Suiko et al., 2017). Regarding fish species, the SULT genes have been investigated mainly in zebrafish, and 20 distinct paralogs (9 SULT1s, 3 SULT2s, 5 SULT3s, 1 SULT4, 1 SULT5, and 1 SULT6) have been characterized. Among these, SULT1ST6, one of the nine SULT1 paralogs, displays strict substrate specificity for estrogens (e.g., estrone). This gene is thought to be involved in organogenesis, such as the development of the eye and muscle in zebrafish (Yasuda et al., 2005).

On the other hand, there is no report about the relationship between estrogen sulfotransferase and sex development. In fish, steroid hormones are considered to be the key factor for sex determination

379 (Yamamoto, 1969; Devlin and Nagahama, 2002; Nakamura, 2010), wherein sex can change

380 depending on the levels of estrogen or androgen production in gonad cells. The gene directly contributing to steroidogenesis has also been reported as a sex-determination gene in yellowtails (Koyama et al., 2019). Since endogenous estrogens are inactivated by sulfation (Raftogianis et al., 2000), it may not be surprising that the function of sult1st6 is involved in sex development. If this gene is expressed in gonad cells at the initiation of sex development, it may inhibit female development by depleting active estrogens, resulting in male development. In the present study, we found that sult1st6y of PBT was paternally inherited in the population. It is unlikely that an 
391 sult1st6a was close to the artifact GWA peak. This observation is not only attributable to

392 cross-mapping, as mentioned before, but also indirectly suggests that the sult1st6-like gene might be

393 associated with the sex of PBT. Since sult1st6a is inherited regardless of sex in a diploid manner, we

394 speculate that sult1st6a plays a common role in PBT males and females, for example, in

395 organogenesis at the early developmental stage, as proposed in the model fish. This suggests that

396 sult st6y could have been immune from the original role of sult1st6 as a redundant copy: after gene

397 duplication in the ancestor of PBT, the function of sult1 st6y may have been spatiotemporally

398 differentiated from that of sult1 st6a in the proto-paternal line. In fact, it has been reported that a

399 copy of duplicated genes may be sex-determination gene in fishes (Hattori et al., 2012; Yano et al.,

400 2012; Matsuda and Sakaizumi, 2016). We found that homologs of sult1st6y and sult1st6a were

401 present in the southern bluefin tuna. Phylogenetic analysis suggested that gene duplication occurred

402 in the common ancestor of PBT and SBT. The duplication event is specific to the tuna lineage, not

403 observed in other fishes, except for tandem duplications in some species. According to the

404 transcriptome, SBT sult1st6y was expressed in male's testis but not in female's ovary. Considering

405 that the expression of sult1st6a was at least detectable in females, although at a much lower level

406 than in males, sult1st6y below the detection limit might be originally absent from SBT females like

407 PBT females. Although this hypothesis will need to be tested by sequencing the entire SBT genome,

408 our observations suggest that SBT sult1st6y may also be involved in sex development like PBT

409 sult1st6y.

410 The results presented in this study provide an attractive hypothesis for sult1st6y being a

411 sex-determination gene in Thunnus fishes, or at least functioning at an important point in the

412 sex-differentiation cascade. Our hypothesis may be tested by further genetic experiments, although

413 the handling of tuna in tanks is not easy. Recently, genome-editing technology has been applied to

414 the mutagenesis of tuna (Higuchi et al., 2019). For example, it may be possible to check whether the 
415 sex ratio of larvae is heavily biased by the knockout of sult1st6y in fertilized eggs. Lastly, it is worth

416 emphasizing that the CNV-based approach used in this study is very useful for identifying a

417 trait-associated region using short reads when it is involved in large structural variants. In particular,

418 when the region contains recently duplicated sequences, the traditional SNV-based approach may not

419 only miss structural variants but also produce artifacts due to cross-mapping to unassociated regions.

420 In other fish species, sex-associated SNVs have often been observed in multiple scaffolds (Fowler

421 and Buonaccorsi, 2016; Star et al., 2016; Dong et al., 2019), some of which might be cases of

422 cross-mapping of short reads, unless the sexes are polygenically determined. We believe that a

423 combination of traditional SNV- and CNV-based approaches is useful for the identification of

424 sex-associated genes in fish species with a genetic sex-determination system. 
Data availability statement

426 The genome sequences of Pacific bluefin tuna obtained in this study were deposited to the DNA

427 Data Bank of Japan. The accession numbers can be found in the article.

428

429 Author contributions

$430 \mathrm{YN}$ and AF conceived the study. KG coordinated the experiments for sampling, and KH, KK, MY,

431 and TT performed those. AF conducted genome sequencing, and YN conducted bioinformatic

432 analyses. YN drafted the manuscript, and MY helped to improve it. All authors read and approved

433 the final version.

434

435 Acknowledgements

436 This work was supported by the Japan Fisheries Research and Education Agency (No. 3BA101). We

437 would like to thank Editage (www.editage.jp) for English language editing. 


\section{References}

Agawa, Y., Iwaki, M., Komiya, T., Honryo, T., Tamura, K., Okada, T., et al. (2015). Identification of male sex-linked DNA sequence of the cultured Pacific bluefin tuna Thunnus orientalis. Fisheries Science 81(1), 113-121. doi: 10.1007/s12562-014-0833-8.

Aken, B.L., Achuthan, P., Akanni, W., Amode, M.R., Bernsdorff, F., Bhai, J., et al. (2017). Ensembl 2017. Nucleic Acids Res 45(D1), D635-D642. doi: 10.1093/nar/gkw1104.

Altschul, S.F., Gish, W., Miller, W., Myers, E.W., and Lipman, D.J. (1990). Basic local alignment search tool. $J$ Mol Biol 215(3), 403-410. doi: 10.1016/S0022-2836(05)80360-2

S0022-2836(05) 80360-2 [pii].

Altschul, S.F., Madden, T.L., Schaffer, A.A., Zhang, J., Zhang, Z., Miller, W., et al. (1997). Gapped BLAST and PSI-BLAST: a new generation of protein database search programs. Nucleic Acids Res 25(17), 3389-3402. doi: gka562 [pii].

Bar, I., Cummins, S., and Elizur, A. (2016). Transcriptome analysis reveals differentially expressed genes associated with germ cell and gonad development in the Southern bluefin tuna (Thunnus maccoyii). BMC Genomics 17, 217. doi: 10.1186/s12864-016-2397-8.

Bolger, A.M., Lohse, M., and Usadel, B. (2014). Trimmomatic: a flexible trimmer for Illumina sequence data. Bioinformatics 30(15), 2114-2120. doi: 10.1093/bioinformatics/btu170.

Chin, C.S., Alexander, D.H., Marks, P., Klammer, A.A., Drake, J., Heiner, C., et al. (2013). Nonhybrid, finished microbial genome assemblies from long-read SMRT sequencing data. Nat Methods 10(6), 563-569. doi: 10.1038/nmeth.2474.

Coombe, L., Zhang, J., Vandervalk, B.P., Chu, J., Jackman, S.D., Birol, I., et al. (2018). ARKS: chromosome-scale scaffolding of human genome drafts with linked read kmers. BMC Bioinformatics 19(1), 234. doi: 10.1186/s12859-018-2243-x.

Cui, Z., Liu, Y., Wang, W., Wang, Q., Zhang, N., Lin, F., et al. (2017). Genome editing reveals dmrt1 as an essential male sex-determining gene in Chinese tongue sole (Cynoglossus semilaevis). Sci Rep 7, 42213. doi: 10.1038/srep42213.

Devlin, R.H., and Nagahama, Y. (2002). Sex determination and sex differentiation in fish: an overview of genetic, physiological, and environmental influences. Aquaculture 208(3), 191-364. doi: https://doi.org/10.1016/S0044-8486(02)00057-1.

Dong, C., Jiang, P., Zhang, J., Li, X., Li, S., Bai, J., et al. (2019). High-Density Linkage Map and Mapping for Sex and Growth-Related Traits of Largemouth Bass (Micropterus salmoides). Front Genet 10, 960. doi: 10.3389/fgene.2019.00960. 
Endelman, J.B. (2011). Ridge Regression and Other Kernels for Genomic Selection with R Package rrBLUP. The Plant Genome 4(3), 250-255. doi: 10.3835/plantgenome2011.08.0024.

Fowler, B.L., and Buonaccorsi, V.P. (2016). Genomic characterization of sex-identification markers in Sebastes carnatus and Sebastes chrysomelas rockfishes. Mol Ecol 25(10), 2165-2175. doi: 10.1111/mec. 13594 .

Gonzalez-Dominguez, J., and Schmidt, B. (2016). ParDRe: faster parallel duplicated reads removal tool for sequencing studies. Bioinformatics 32(10), 1562-1564. doi: 10.1093/bioinformatics/btw038.

Haas, B.J., Papanicolaou, A, Yassour, M., Grabherr, M., Blood, P.D., Bowden, J., et al. (2013). De novo transcript sequence reconstruction from RNA-seq using the Trinity platform for reference generation and analysis. Nat Protoc 8(8), 1494-1512. doi: 10.1038/nprot.2013.084.

Hattori, R.S., Murai, Y., Oura, M., Masuda, S., Majhi, S.K., Sakamoto, T., et al. (2012). A Y-linked anti-Mullerian hormone duplication takes over a critical role in sex determination. Proc Natl Acad Sci $U$ S A 109(8), 2955-2959. doi: 10.1073/pnas.1018392109.

Higuchi, K., Kazeto, Y., Ozaki, Y., Yamaguchi, T., Shimada, Y., Ina, Y., et al. (2019). Targeted mutagenesis of the ryanodine receptor by Platinum TALENs causes slow swimming behaviour in Pacific bluefin tuna (Thunnus orientalis). Sci Rep 9(1), 13871. doi: 10.1038/s41598-019-50418-3.

Jackman, S.D., Coombe, L., Chu, J., Warren, R.L., Vandervalk, B.P., Yeo, S., et al. (2018). Tigmint: correcting assembly errors using linked reads from large molecules. $B M C$ Bioinformatics 19(1), 393. doi: 10.1186/s12859-018-2425-6.

Kamiya, T., Kai, W., Tasumi, S., Oka, A., Matsunaga, T., Mizuno, N., et al. (2012). A trans-species missense SNP in Amhr2 is associated with sex determination in the tiger pufferfish, Takifugu rubripes (fugu). PLoS Genet 8(7), e1002798. doi: 10.1371/journal.pgen.1002798.

Katoh, K., Asimenos, G., and Toh, H. (2009). Multiple alignment of DNA sequences with MAFFT. Methods Mol Biol537, 39-64. doi: 10.1007/978-1-59745-251-9_3.

Koyama, T., Nakamoto, M., Morishima, K., Yamashita, R., Yamashita, T., Sasaki, K., et al. (2019). A SNP in a steroidogenic enzyme is associated with phenotypic sex in Seriola fishes. Curr Biol 29(11), 1901-1909 e1908. doi: 10.1016/j.cub.2019.04.069.

Kozlov, A.M., Darriba, D., Flouri, T., Morel, B., and Stamatakis, A. (2019). RAxML-NG: a fast, scalable and user-friendly tool for maximum likelihood phylogenetic inference. Bioinformatics 35(21), 4453-4455. doi: 10.1093/bioinformatics/btz305. 
Li, B., and Dewey, C.N. (2011). RSEM: accurate transcript quantification from RNA-Seq data with or without a reference genome. BMC Bioinformatics 12, 323. doi: 10.1186/1471-2105-12-323.

Li, H., Handsaker, B., Wysoker, A., Fennell, T., Ruan, J., Homer, N., et al. (2009). The Sequence Alignment/Map format and SAMtools. Bioinformatics 25(16), 2078-2079. doi: 10.1093/bioinformatics/btp352.

Lin, H.-N., and Hsu, W.-L. (2019). MapCaller - An integrated and efficient tool for short-read mapping and variant calling using high-throughput sequenced data. bioRxiv, 783605. doi: 10.1101/783605.

Marcais, G., Delcher, A.L., Phillippy, A.M., Coston, R., Salzberg, S.L., and Zimin, A. (2018). MUMmer4: A fast and versatile genome alignment system. PLoS Comput Biol14(1), e1005944. doi: 10.1371/journal.pcbi.1005944.

Matsuda, M., Nagahama, Y., Shinomiya, A., Sato, T., Matsuda, C., Kobayashi, T., et al. (2002). DMY is a Y-specific DM-domain gene required for male development in the medaka fish. Nature 417(6888), 559-563. doi: 10.1038/nature751.

Matsuda, M., and Sakaizumi, M. (2016). Evolution of the sex-determining gene in the teleostean genus Oryzias. Gen Comp Endocrinol 239, 80-88. doi: 10.1016/j.ygcen.2015.10.004.

Nakamura, M. (2010). The mechanism of sex determination in vertebrates-are sex steroids the key-factor? JExp Zool A Ecol Genet Physiol 313(7), 381-398. doi: 10.1002/jez.616.

Nakamura, Y., Mori, K., Saitoh, K., Oshima, K., Mekuchi, M., Sugaya, T., et al. (2013). Evolutionary changes of multiple visual pigment genes in the complete genome of Pacific bluefin tuna. Proc Natl Acad Sci U S A 110(27), 11061-11066. doi: 10.1073/pnas.1302051110.

Nanda, I., Kondo, M., Hornung, U., Asakawa, S., Winkler, C., Shimizu, A., et al. (2002). A duplicated copy of DMRT1 in the sex-determining region of the Y chromosome of the medaka, Oryzias latipes. Proc Natl Acad Sci U SA 99(18), 11778-11783. doi: 10.1073/pnas.182314699.

Nowotny, M. (2009). Retroviral integrase superfamily: the structural perspective. EMBO $\operatorname{Rep~10(2),~144-151.~doi:~10.1038/embor.2008.256.~}$

Pan, Q., Feron, R., Yano, A., Guyomard, R., Jouanno, E., Vigouroux, E., et al. (2019). Identification of the master sex determining gene in Northern pike (ESox lucius) reveals restricted sex chromosome differentiation. PLoS Genet 15(8), e1008013. doi: 10.1371/journal.pgen.1008013.

545 Pennell, M.W., Mank, J.E., and Peichel, C.L. (2018). Transitions in sex determination and 
sex chromosomes across vertebrate species. Mol Ecol 27(19), 3950-3963. doi: 10.1111/mec. 14540 .

Raftogianis, R., Creveling, C., Weinshilboum, R., and Weisz, J. (2000). Estrogen metabolism by conjugation. $J$ Natl Cancer Inst Monogr (27), 113-124. doi: 10.1093/oxfordjournals.jncimonographs.a024234.

Simão, F.A., Waterhouse, R.M., Ioannidis, P., Kriventseva, E.V., and Zdobnov, E.M. (2015). BUSCO: assessing genome assembly and annotation completeness with single-copy orthologs. Bioinformatics 31(19), 3210-3212. doi: 10.1093/bioinformatics/btv351.

Slater, G.S., and Birney, E. (2005). Automated generation of heuristics for biological sequence comparison. BMC Bioinformatics 6, 31. doi: 1471-2105-6-31 [pii]

10.1186/1471-2105-6-31.

Star, B., Torresen, O.K., Nederbragt, A.J., Jakobsen, K.S., Pampoulie, C., and Jentoft, S. (2016). Genomic characterization of the Atlantic cod sex-locus. Sci Rep 6, 31235. doi: 10.1038/srep31235.

Suda, A., Nishiki, I., Iwasaki, Y., Matsuura, A., Akita, T., Suzuki, N., et al. (2019). Improvement of the Pacific bluefin tuna (Thunnus orientalis) reference genome and development of male-specific DNA markers. Sci Rep 9(1), 14450. doi: 10.1038/s41598-019-50978-4.

Suiko, M., Kurogi, K., Hashiguchi, T., Sakakibara, Y., and Liu, M.C. (2017). Updated perspectives on the cytosolic sulfotransferases (SULTs) and SULT-mediated sulfation. Biosci Biotechnol Biochem 81(1), 63-72. doi: 10.1080/09168451.2016.1222266.

Walker, B.J., Abeel, T., Shea, T., Priest, M., Abouelliel, A., Sakthikumar, S., et al. (2014). Pilon: an integrated tool for comprehensive microbial variant detection and genome assembly improvement. PLoS One 9(11), e112963. doi: 10.1371/journal.pone.0112963.

Wang, X., Zheng, Z., Cai, Y., Chen, T., Li, C., Fu, W., et al. (2017). CNVcaller: highly efficient and widely applicable software for detecting copy number variations in large populations. Gigascience 6(12), 1-12. doi: 10.1093/gigascience/gix115.

Warren, R.L., Yang, C., Vandervalk, B.P., Behsaz, B., Lagman, A., Jones, S.J., et al. (2015). LINKS: Scalable, alignment-free scaffolding of draft genomes with long reads. Gigascience 4, 35. doi: 10.1186/s13742-015-0076-3.

Weisenfeld, N.I., Kumar, V., Shah, P., Church, D.M., and Jaffe, D.B. (2017). Direct determination of diploid genome sequences. Genome Res 27(5), 757-767. doi: 10.1101/gr.214874.116.

Yamamoto, T. (1969). "Sex Differentiation," in Fish Physiology, eds. W.S. Hoar \& D.J. 
Randall. Academic Press), 117-175.

Yano, A., Guyomard, R., Nicol, B., Jouanno, E., Quillet, E., Klopp, C., et al. (2012). An immune-related gene evolved into the master sex-determining gene in rainbow trout, Oncorhynchus mykiss. Curr Biol 22(15), 1423-1428. doi: 10.1016/j.cub.2012.05.045.

Yasuda, S., Liu, C.C., Takahashi, S., Suiko, M., Chen, L., Snow, R., et al. (2005). Identification of a novel estrogen-sulfating cytosolic SULT from zebrafish: molecular cloning, expression, characterization, and ontogeny study. Biochem Biophys Res Commun 330(1), 219-225. doi: 10.1016/j.bbrc.2005.02.152.

Yazawa, R., Takeuchi, Y., Machida, Y., Amezawa, K., Kabeya, N., Tani, R., et al. (2019). Production of triploid eastern little tuna, Euthynnus affinis (Cantor, 1849). 


\section{Tables}

Table 1. Assembly statistics of PBT genomes

\begin{tabular}{lll}
\hline & This study & Previous study* \\
\hline Number of scaffolds & 948 & 444 \\
Total scaffold size (Mbp) & 827 & 787 \\
N50 scaffold size (Mbp) & 13.2 & 7.9 \\
BUSCO completeness (\%) & 96.3 & 89.3 \\
Single copy BUSCOs (\%) & 93.6 & 86.1 \\
Duplicated BUSCOs $(\%)$ & 2.7 & 3.2 \\
\hline
\end{tabular}

*Accession no. BKCK00000000. 
bioRxiv preprint doi: https://doi.org/10.1101/2021.06.15.448492; this version posted June 29, 2021. The copyright holder for this preprint (which was not certified by peer review) is the author/funder. All rights reserved. No reuse allowed without permission.

596 Table 2. Amplified sizes of sex identification PCR primer sets

Amplified size in the

\begin{tabular}{llllll}
\multicolumn{2}{l}{ Primer set } & \multicolumn{2}{l}{ This study } & & \multicolumn{2}{l}{ previous study (bp) } \\
\cline { 2 - 3 } \cline { 5 - 6 } & M44 & M175 & & Female & Male \\
\hline scaf64_3724604_F/R & - & 113 & & - & 113 \\
scaf64_3726411_F/R & - & 143 & & - & 143 \\
scaf64_3724591_F/R & 149 & 142 & & 149 & 142,149 \\
\hline
\end{tabular}

597 


\section{$598 \quad$ Figure legends}

599

600 Figure 1. Sequence comparison between PBT male scaffolds M175 (X-axis) and M44 (Y-axis). Left

601 panel indicates the full-size comparison (232,647 bp vs. 4,583,724 bp), and the boxed region is

602 enlarged at right panel. In the right panel, $\mathrm{SLR}_{\mathrm{M} 44}$ and $\mathrm{SLR}_{\mathrm{M} 175}$ are highlighted in gray.

603

604 Figure 2. Sequence comparison between male scaffold M44 and female scaffold F64. The 6.5-kb

605 SLRs are indicated with arrows, respectively.

607 Figure 3. Mapped read depths around SLRs in resequenced PBT samples. For each of the female

608 (DRR177382) and male (DRR177383) samples, SLR is highlighted in gray. The read depths for all

609 the 31 samples are shown in Supplementary figure 2.

610

611 Figure 4. Comparison of relative read depths between males and females for $\mathrm{SLR}_{\mathrm{M} 44} / \mathrm{SLR}_{\mathrm{M} 175}$. The

612 mapping of resequenced reads was conducted using an unmasked male reference.

613

614 Figure 5. Genome-wide association with the sex of PBT using the female reference sequences. For

615 the unmasked (A and C) and masked (B and D) references, Manhattan plot and Q-Q plot are shown,

616 respectively. In Manhattan plots, genome-wide significance cutoffs based on Bonferroni correction

617 are indicate by a red line, and the significant peak in scaffold F64 is indicated by a triangle. $P$-values

618 lower than $10^{-15}$ are treated as $10^{-15}$ for convenience.

619

620 Figure 6. Classification of the resequenced reads mapped to $\mathrm{SLR}_{\mathrm{F} 64}$ in the female genome. 
622 Figure 7. Genome-wide association with copy number between PBT males and females. The

623 mapping of resequenced reads was conducted using a masked male reference. The plots on scaffold

624 M175 are indicated by triangles. (A) Differences in regional copy number between males and

625 females are denoted as the values of $-\log _{10}(p)$ in the Mann-Whitney $U$ test. Upward plots indicate

626 that the copy number is larger in males than in females, while downward plots indicate the inverse.

627 Genome-wide significance cutoffs based on Bonferroni correction are indicated by dashed lines. (B)

628 Manhattan plot (left) and Q-Q plot (right) from CNV-based GWA scan. The regions with copy

629 number $0,0.5$, or 1 were selected $\left(P\right.$-values lower than $10^{-15}$ were treated as $\left.10^{-15}\right)$. In the Manhattan

630 plot, genome-wide significance cutoff based on Bonferroni correction is indicated by a red line.

632 Figure 8. Protein-coding regions in scaffold M175. The region predicted with a copy number of 0.5

633 and 10.7-kb SLR M175 $_{1}$ are shown in red and black, respectively.

635 Figure 9. Phylogenetic tree of sult1 st6 in fish species. For each of the nodes, the bootstrap

636 probability is indicated when it is $>95 \%$. The tree constructed using more homologs linked with the

637 Ensembl IDs is shown in Supplementary Figure 5.

639 Figure 10. Expression of sult1st6 in southern bluefin tuna's gonad cells. (A) sult1st6a and (B)

640 sult1st6y. 


\section{Figures}

Fig. 1

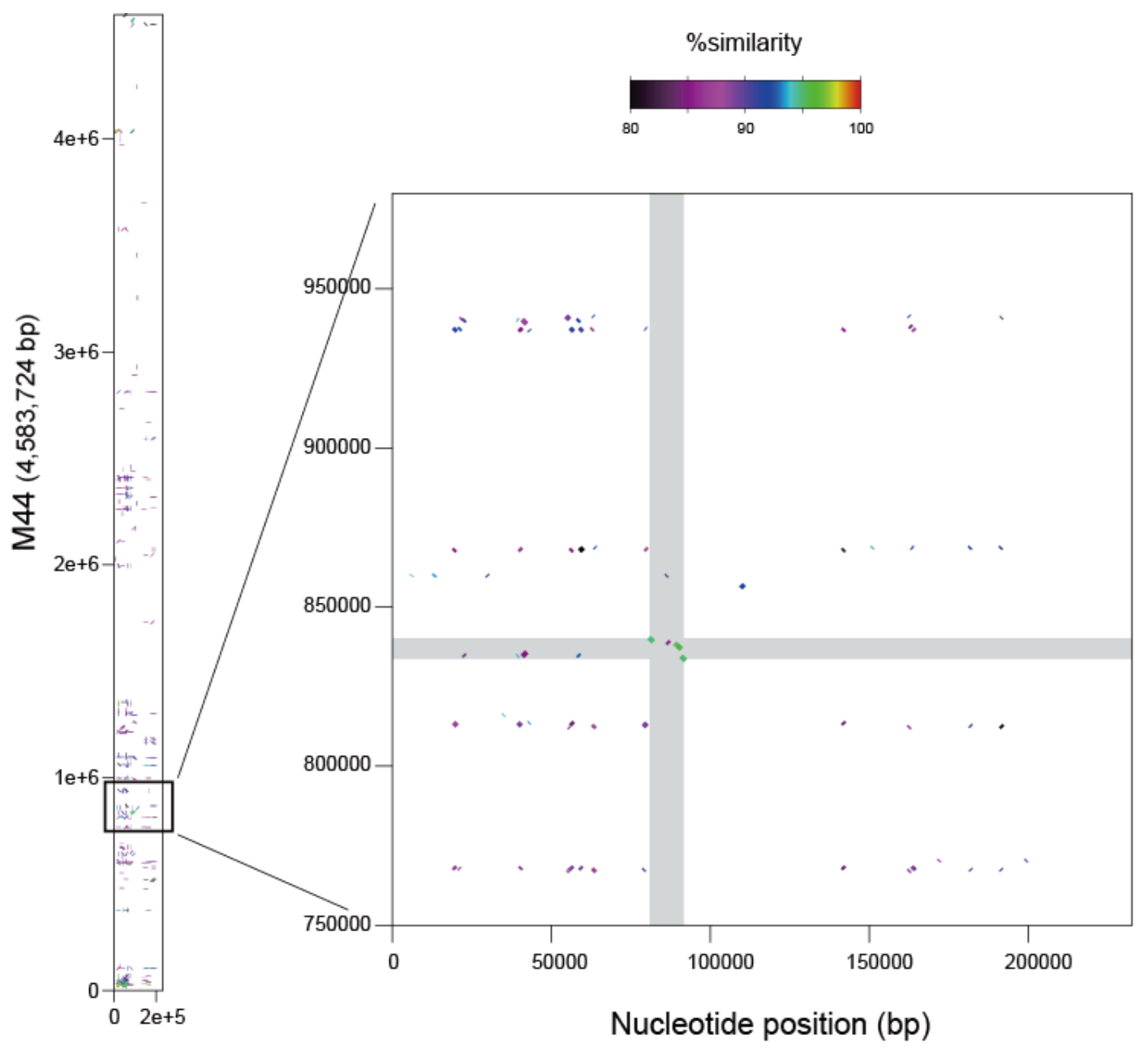

M175 (232,647 bp) 
bioRxiv preprint doi: https://doi.org/10.1101/2021.06.15.448492; this version posted June 29, 2021. The copyright holder for this preprint (which was not certified by peer review) is the author/funder. All rights reserved. No reuse allowed without permission.

Fig. 2

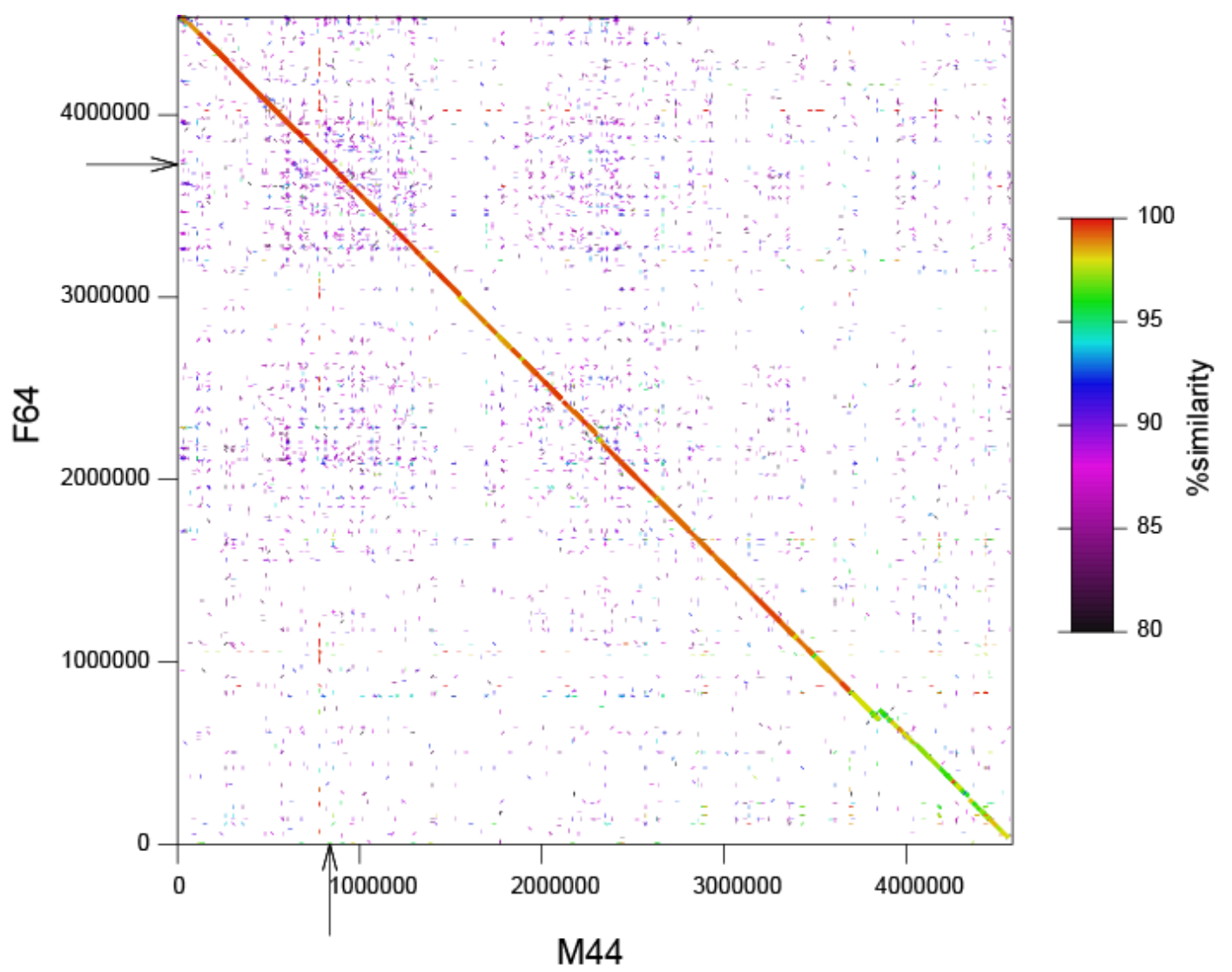


Fig. 3
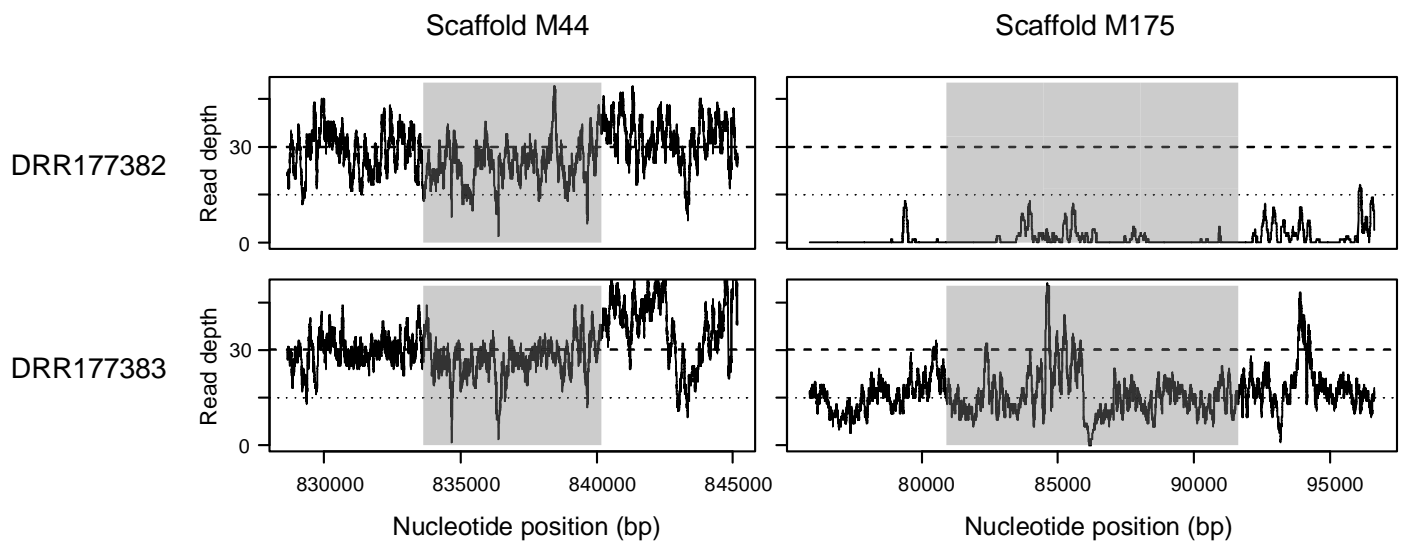
Fig. 4

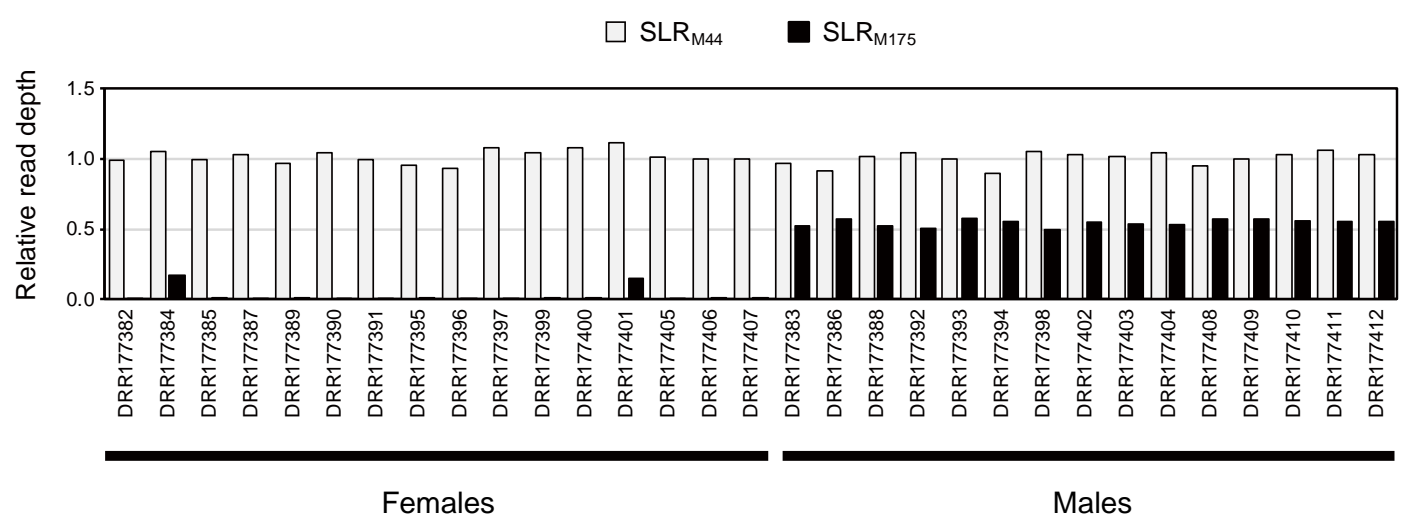


Fig. 5

A

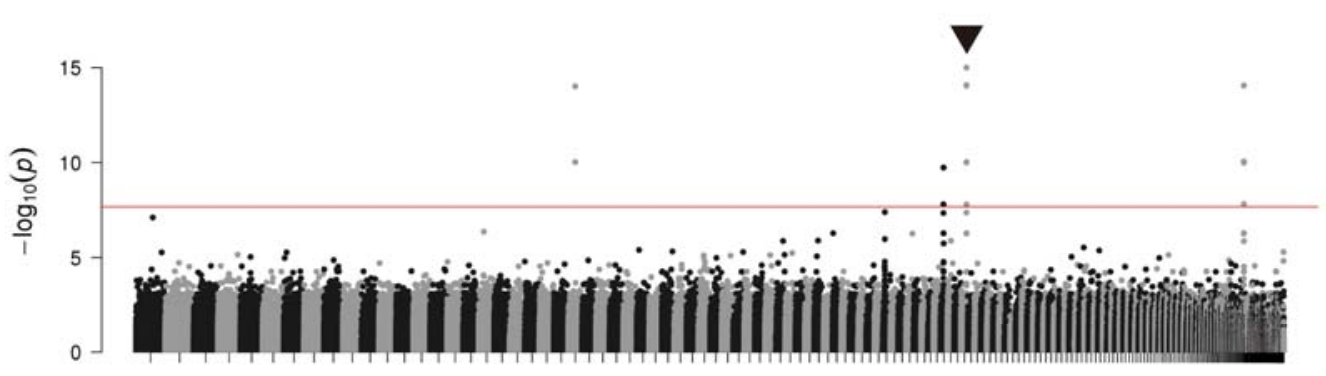

B

Scaffold (F1, F2, F3, ...)

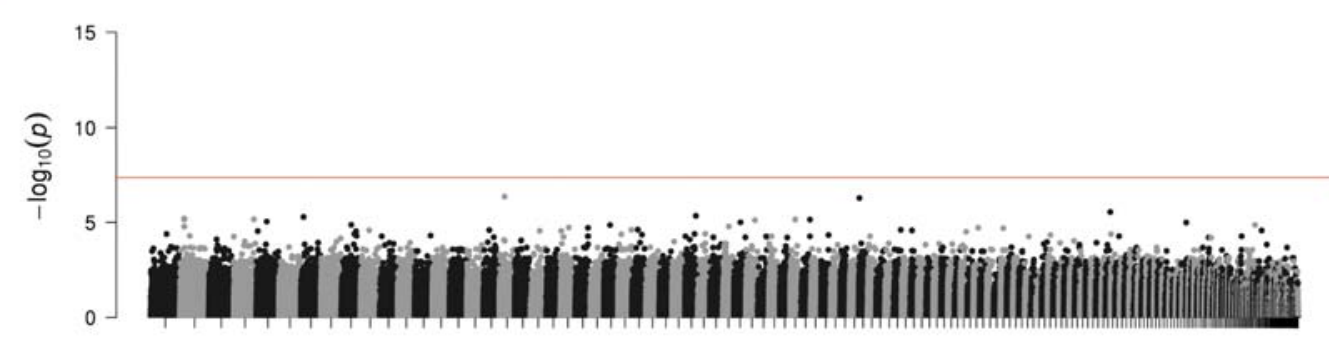

Scaffold (F1, F2, F3, ...)

C

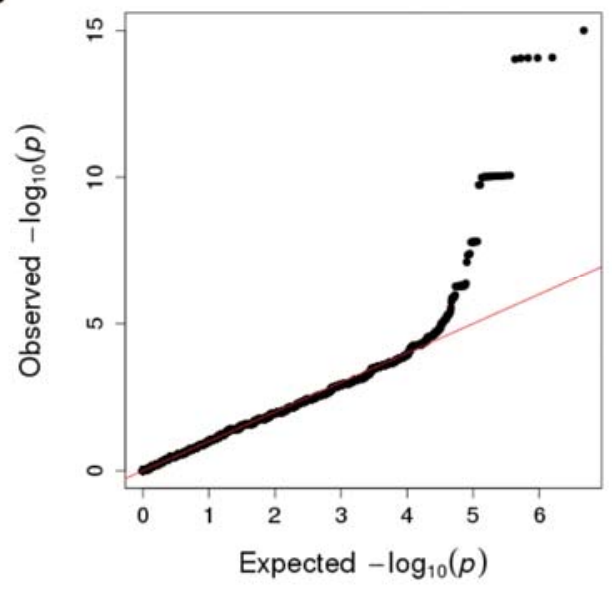

D

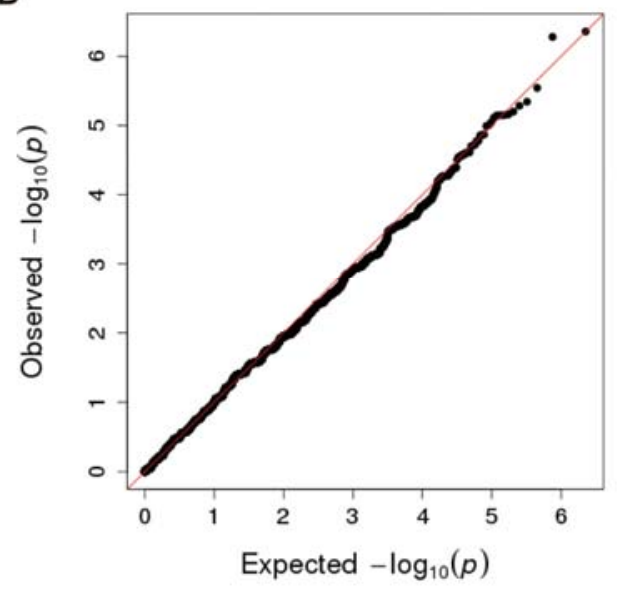


Fig. 6

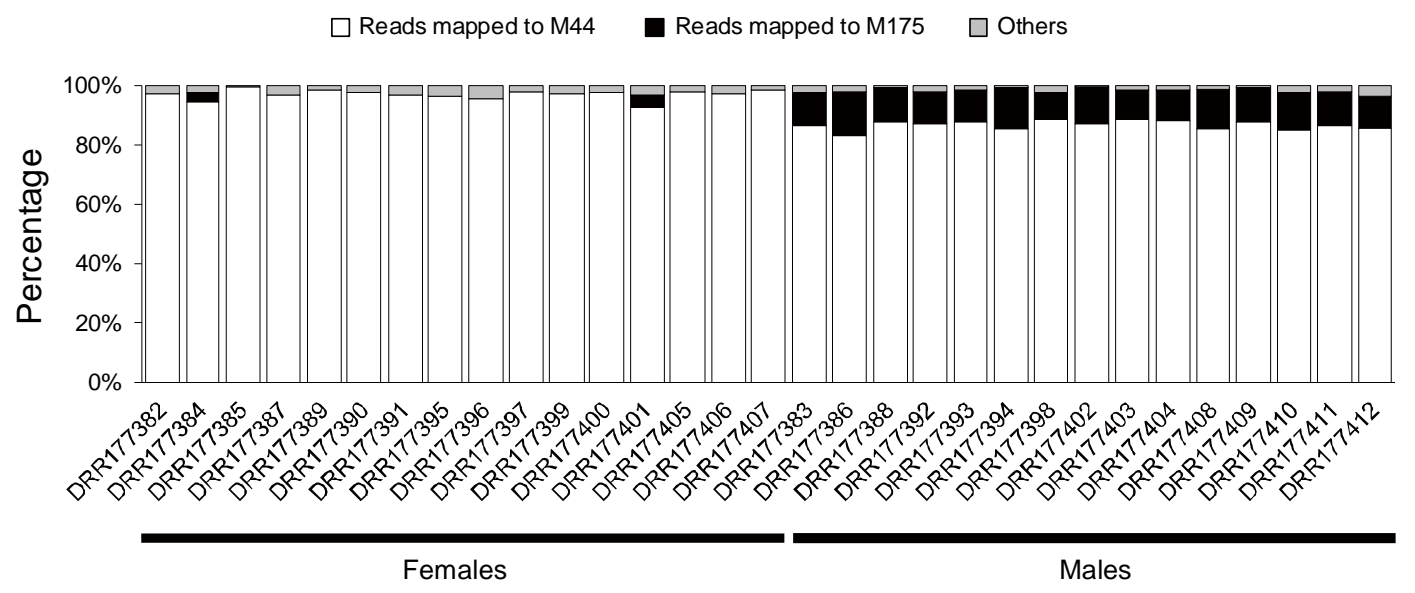


bioRxiv preprint doi: https://doi.org/10.1101/2021.06.15.448492; this version posted June 29, 2021. The copyright holder for this preprint (which was not certified by peer review) is the author/funder. All rights reserved. No reuse allowed without permission.

Fig. 7

A

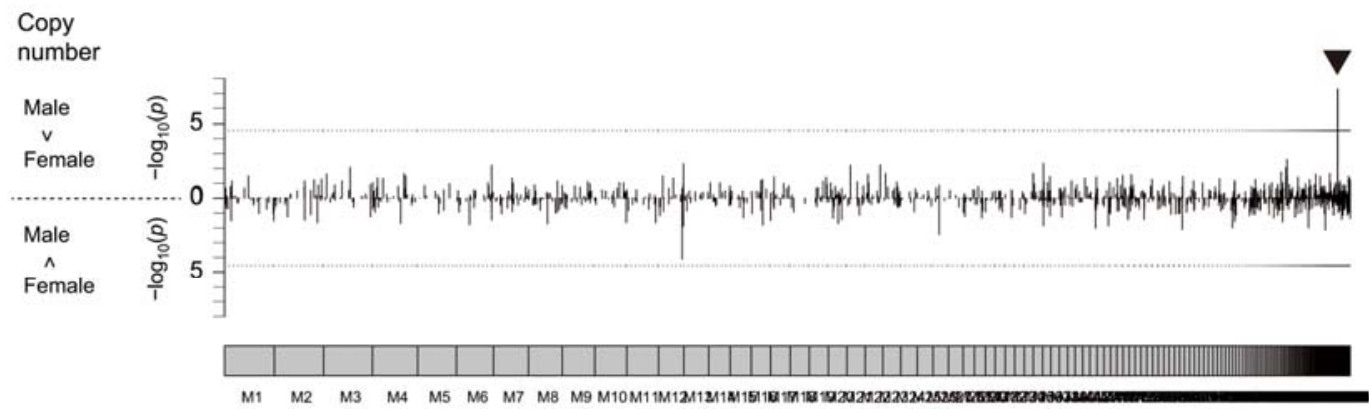

Scaffold (M1, M2, M3, ...)

B
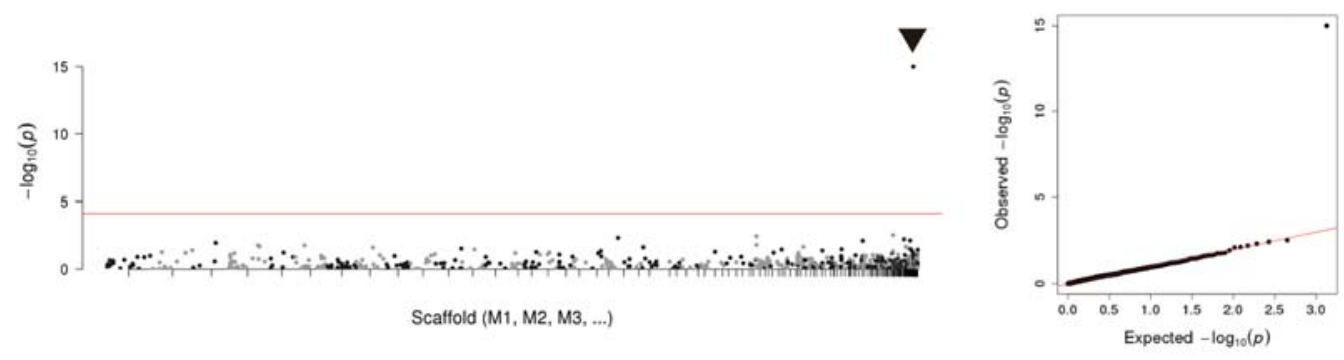

Scaffold (M1, M2, M3, ...) 
bioRxiv preprint dol: https://doi.org/10.1101/2021.06.15.448492; this version posted June 29, 2021. The copyright holder for this preprint (which was not certified by peer review) is the author/funder. All rights reserved. No reuse allowed without permission.

Fig. 8

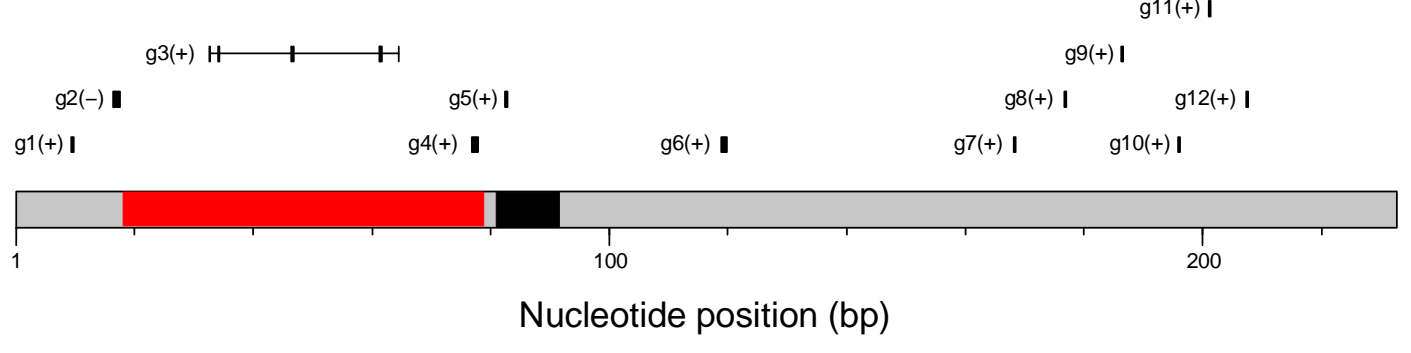


Fig. 9

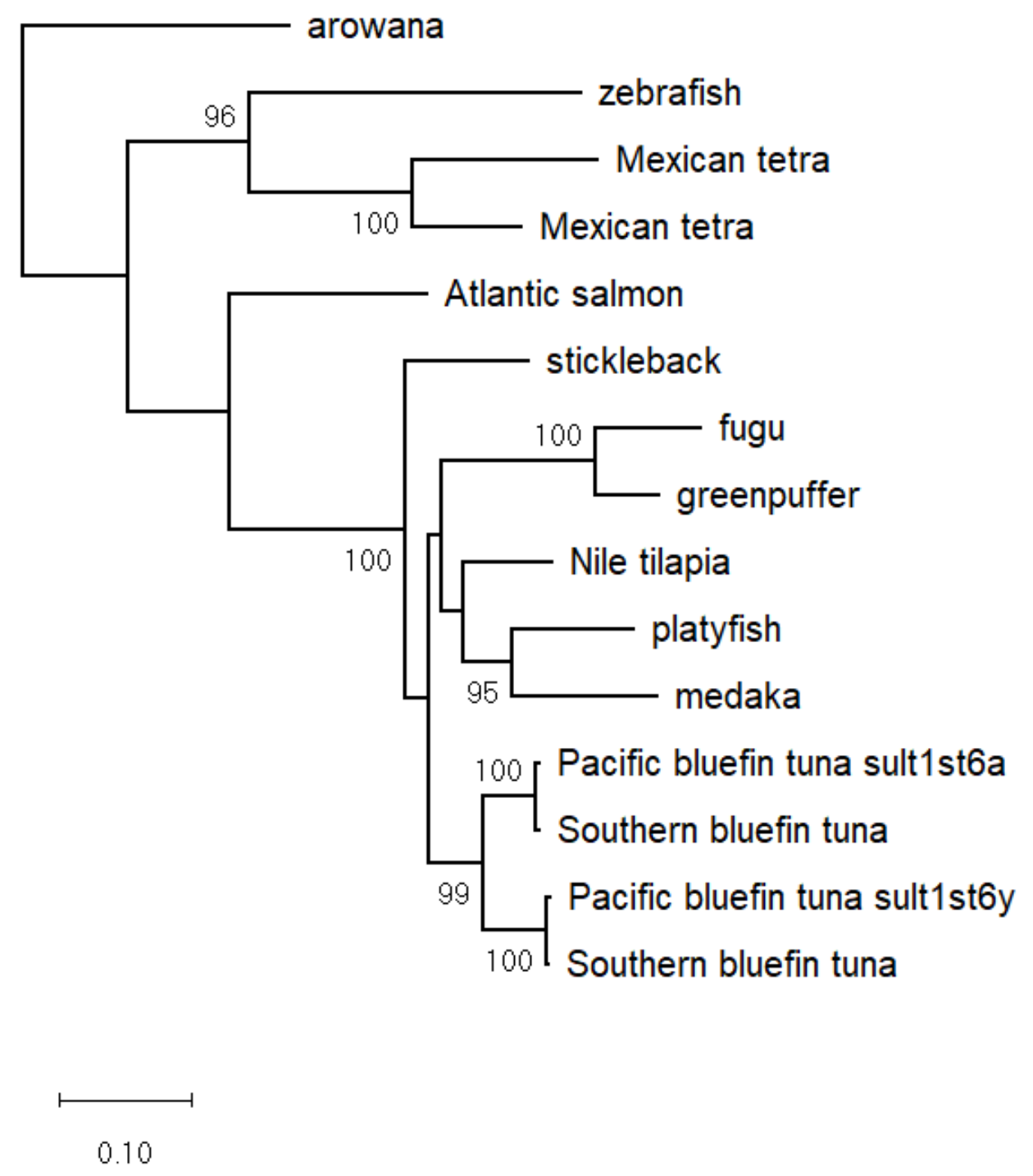


Fig. 10

\section{A}

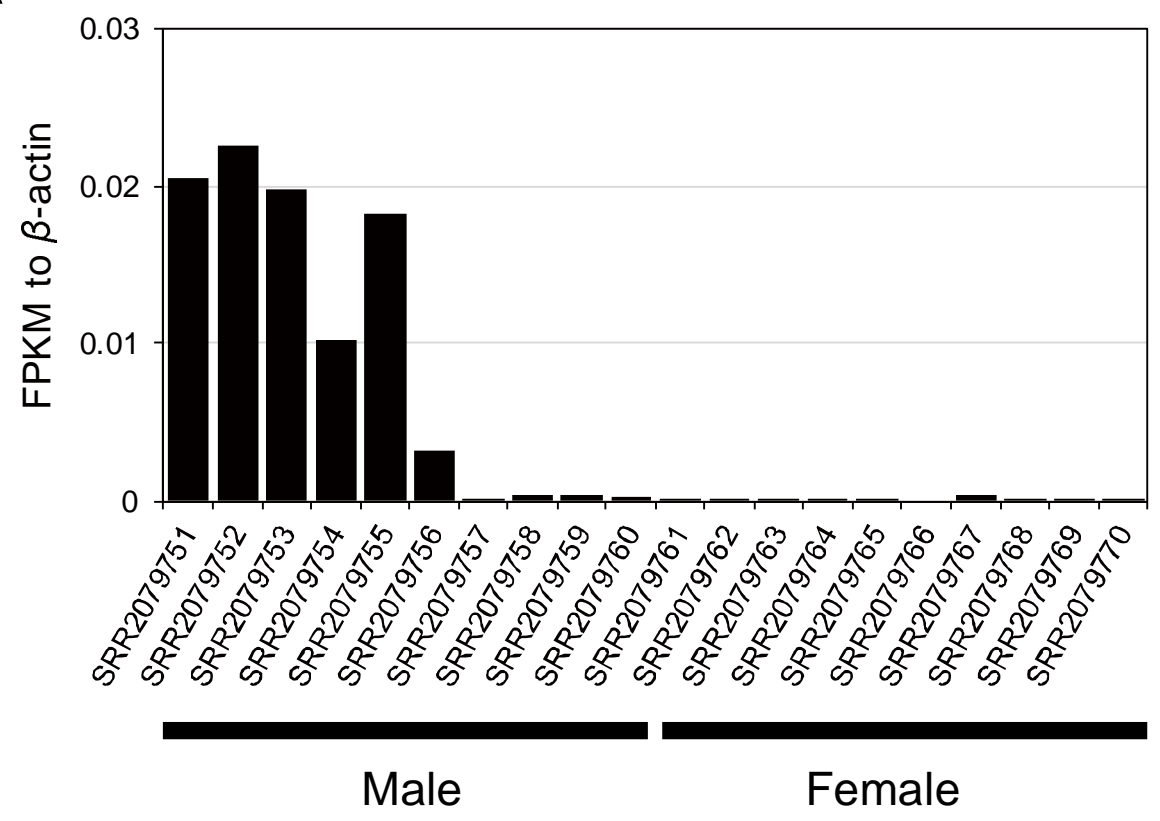

B

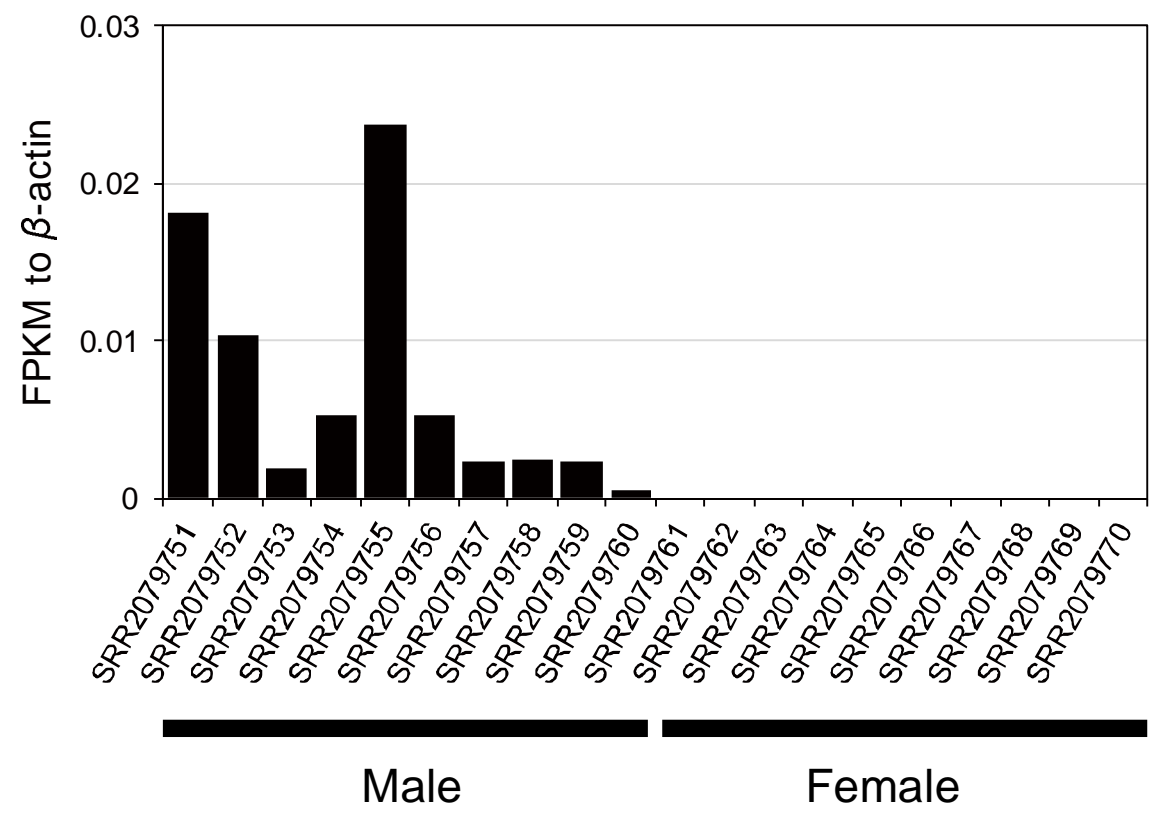

